\title{
Mucormycosis in COVID-19 pandemic: study at tertiary hospital in India
}

\author{
Reshma P. Chavan ${ }^{1}$ (D) Shivraj M. Ingole ${ }^{2} \cdot$ Hamna Abdul Nazir ${ }^{1} \cdot$ Wilson V. Desai ${ }^{1}$. Gajanan S. Kanchewad ${ }^{1}$
}

Received: 12 December 2021 / Accepted: 21 January 2022 / Published online: 5 February 2022

(c) The Author(s), under exclusive licence to Springer-Verlag GmbH Germany, part of Springer Nature 2022

\begin{abstract}
Aim During the second wave of COVID-19, cases of mucormycosis were increased suddenly over a period of 3 months in Maharashtra, India. An attempt was made to study the clinical profile and risk factors associated with mucormycosis.

Materials and methods A retrospective descriptive study was carried out at a tertiary hospital during May 2021-July 2021. After obtaining informed written consent from the participants, various details of all participants, such as diabetes mellitus, use of steroids in COVID-19 treatment, use of immunosuppressant drugs, oxygen therapy, use of ventilators, complications that occurred during treatment, etc., were noted. All mucormycosis patients were treated with amphotericin B and aggressive surgical treatment.

Results In the present study, $74.7 \%$ of mucormycosis patients were male. $77.4 \%$ of mucormycosis patients were above 40 years of age. $6.7 \%$ of mucormycosis patients were partially vaccinated. Among risk factors, $86.6 \%$ had diabetes mellitus, 84\% had COVID-19 infection, $44 \%$ had received steroids, and $54.7 \%$ had received oxygen. $80 \%$ of patients were present during and within 1 month of COVID-19 infection. 52\% of patients were presented in stage III and $41.3 \%$ were presented in stage II. Despite aggressive surgical debridement along with amphotericin B, mortality was $25.33 \% .5 .3 \%$ of patients had brain abscesses, $8 \%$ of patients had cavernous sinus thrombosis, $4 \%$ of patients had facial nerve palsy and $1.3 \%$ of patients had meningitis.

Conclusion Mucormycosis was predominantly seen in male above the age of 40 years COVID-19 infection and diabetes mellitus was common risk factor for mucormycosis.
\end{abstract}

Keywords COVID-19 $\cdot$ Mucormycosis $\cdot$ Rhino-cerebroorbital $\cdot$ Diabetes, etc.

Reshma P. Chavan

entproblem@gmail.com

Shivraj M. Ingole

drshivraj@gmail.com

Hamna Abdul Nazir

hamnaprov@gmail.com

Wilson V. Desai

wvdesai@gmail.com

Gajanan S. Kanchewad

kanchewadgs@gmail.com

1 Department of ENT, GMC Miraj (Maharashtra), Miraj, India

2 Department of Radiology, GGMC and Sir J.J. Group of Hospitals, Mumbai, Maharashtra, India

\section{Introduction}

Recently, mucormycosis incidence had increased in India due to COVID-19 over a period of 3 months, especially during the second wave of COVID-19 in Maharashtra, India. In India, mucormycosis was seen in 0.14 per 1000 population, which was 80 times higher as compared to developed countries $[1,2]$. As mucormycosis is angio-invasive disease, it causes vascular thrombosis and tissue necrosis [3].

Mucorales spore germinates in people with COVID-19 with low oxygen (hypoxia), high glucose (diabetes, newonset hyperglycaemia, steroid-induced hyperglycaemia), acidic medium (metabolic acidosis, diabetic ketoacidosis), high iron levels (increased ferritins), decreased phagocytic activity of white blood cells due to immunosuppression (SARS-CoV-2 mediated, steroid-mediated or background comorbidities), prolonged hospitalization and mechanical ventilation $[4,5]$. 
Mucormycosis patients present with headache, fever, unilateral facial swelling, orbital cellulitis, palpebral oedema, ptosis, chemosis and ophthalmoplegia. Organ involvement in mucormycosis varies from country to country. In developed countries, lungs were the main site of infection seen in $58.5 \%$, followed by rhinocerebral or rhinoorbital involvement seen in 19.5\% [6]. Study done in an Italian mucormycosis patients, rhino-orbital-cerebral involvement was seen in $35 \%$ and lung involvement was seen in $25 \%$ of cases [7]. In India, the most common type of mucormycosis is the rhino-cerebro-orbital type [8].

In India, diabetes was seen in $8.9 \%$ of the adult population and a total of 77 million populations were affected by diabetes mellitus [9]. Hyperglycaemia was also seen in patients on the glucocorticoids therapy used in severe COVID-19. Diabetes mellitus is associated with impaired neutrophil function, microvascular insufficiency, ketoacidosis, and other metabolic abnormalities. All these conditions were in favour of fungal growth $[4,5]$. Thus, mucormycosis was commonly seen in patients with diabetes mellitus and COVID-19 patients treated with steroids. Although aggressive surgery was done in all patients of mucormycosis, the death rate in mucormycosis remained high.

Mucormycosis of the ethmoid sinus can spread through valveless emissary veins to the cavernous sinus. Maxillary sinus infection can spread to the hard palate and ethmoid sinuses. Infection of the sphenoid sinus can spread to the cavernous sinus. Carotid artery, in the sphenoid sinus gives emboli to the frontal and parietal lobes [10].

The present study aimed to find the risk factors associated with mucormycosis, to study mucormycosis with COVID19 status and to study the complications in mucormycosis patients during treatment.

\section{Materials and methods}

A retrospective descriptive study was carried out at Govt. Medical college Hospital, Miraj (Maharashtra) from May 2021 to July 2021. The study was approved by the Institutional Ethical Committee. A total of 75 patients with mucormycosis were included in the study. All patients included in the present study were microbiology and or histopathology confirmed mucormycosis cases. All mucormycosis patients irrespective of COVID-19 status were included in the study. Admitted patients with an intracranial extension of mucormycosis and later transferred to super speciality centre later were also included in the study.

After obtaining informed written consent from the participating patients, a thorough clinical history was taken regarding diabetes mellitus, use of steroids in COVID-19 treatment, use of immunosuppressant drugs, oxygen therapy, use of ventilators, etc.
Diagnostic endoscopy was done in all patients and samples from the nasal cavity had sent to the microbiology and pathology department for confirmation of diagnosis. $\mathrm{KOH}$ negative and clinically mucormycosis suspect patients were posted in operation theatre for a biopsy and or debridement. The sample was sent to microbiology and histopathology examination for confirmation of the diagnosis of mucormycosis. All patients had a CT or MRI of PNS and brain depending on the presentation of the patient. All patients were done all routine blood investigations.

All mucormycosis patients were treated with amphotericin $\mathrm{B}$ colloidal dispersion (ABCD), liposomal amphotericin $\mathrm{B}$ (L-AMB), amphotericin B lipid complex (ABLC) and amphotericin $B$ conjugated with de-oxycholate (AMBD) depending on the availability of the stock from the government.

The dose of ABCD, L-AMB and ABLC given to patients was $5 \mathrm{mg} / \mathrm{kg} /$ day once daily by intravenous infusion over 4-5 h. The AMB-D was given at $1 \mathrm{mg} / \mathrm{kg} /$ day once daily by intravenous infusion over $4-5 \mathrm{~h}$ with adequate hydration before and after dosing for renal function improvement. The treatment was continued till the cumulative dose of $5 \mathrm{~g}$. Renal function test was done periodically in all patients. In mucormycosis patients with deranged renal function tests, respective amphotericin $B$ was withheld till the renal function tests return to the normal limits.

After anesthetic fitness, patients were posted for aggressive surgical treatment without delay. Postoperative check endoscopy was done weekly and in case of recurrence patients were posted for debridement for second time. Ophthalmology, maxillofacial and neurosurgery consultation was done whenever needed.

Consent for publication of data was taken from every participant in the study.

\section{Results}

\section{Age and sex distribution in the study population}

In the present study, $74.7 \%$ of mucormycosis patients were males and $25.3 \%$ of mucormycosis patients were females (Fig. 1). $77.4 \%$ of mucormycosis patients were above 40 years of age and no patient was under 20 years of age (Fig. 2).

\section{Risk factors associated with mucormycosis}

In the present study, diabetes mellitus was the most common risk factor seen in $86.6 \%$ of patients with mucormycosis. $54.7 \%$ were preexisting diabetes mellitus, $32 \%$ were newly diagnosed diabetes mellitus and in $13.3 \%$ blood sugar levels were in normal range. 
In the present study, $84 \%$ of patients with mucormycosis had a COVID-19 infection. The steroid had received in $44 \%$ of mucormycosis patients. Preexisting diabetes mellitus was

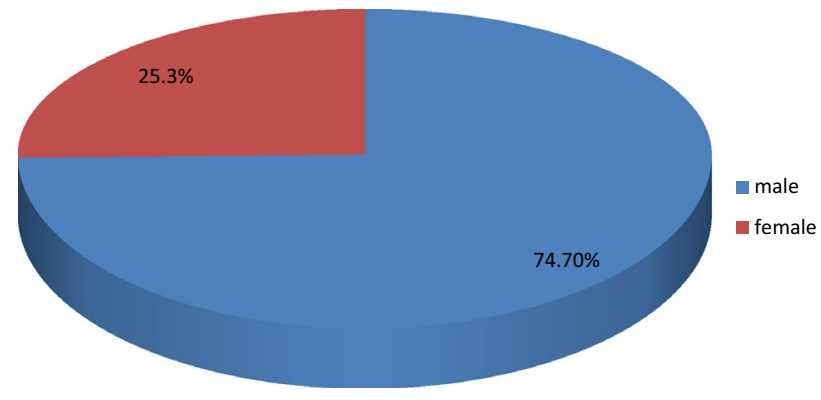

Fig. 1 Sex distribution in study population seen in $24 \%$ patients on steroids. $16 \%$ patients were newly diagnosed diabetes mellitus cases during steroid treatment. $4 \%$ patients were treated with steroids but with blood sugar levels in the normal range.

Oxygen was given in $54.7 \%$ of patients with mucormycosis (Fig. 3).

\section{Gap between COVID-19 treatment and symptoms of mucormycosis}

In the present study, $80 \%$ of patients were present during COVID-19 treatment and within 1 month of COVID-19 infection. No patient with mucormycosis was presented after 3 months of COVID-19 infection (Table 1).
Fig. 2 Age distribution in study population

Fig. 3 Risk factors associated with mucormycosis
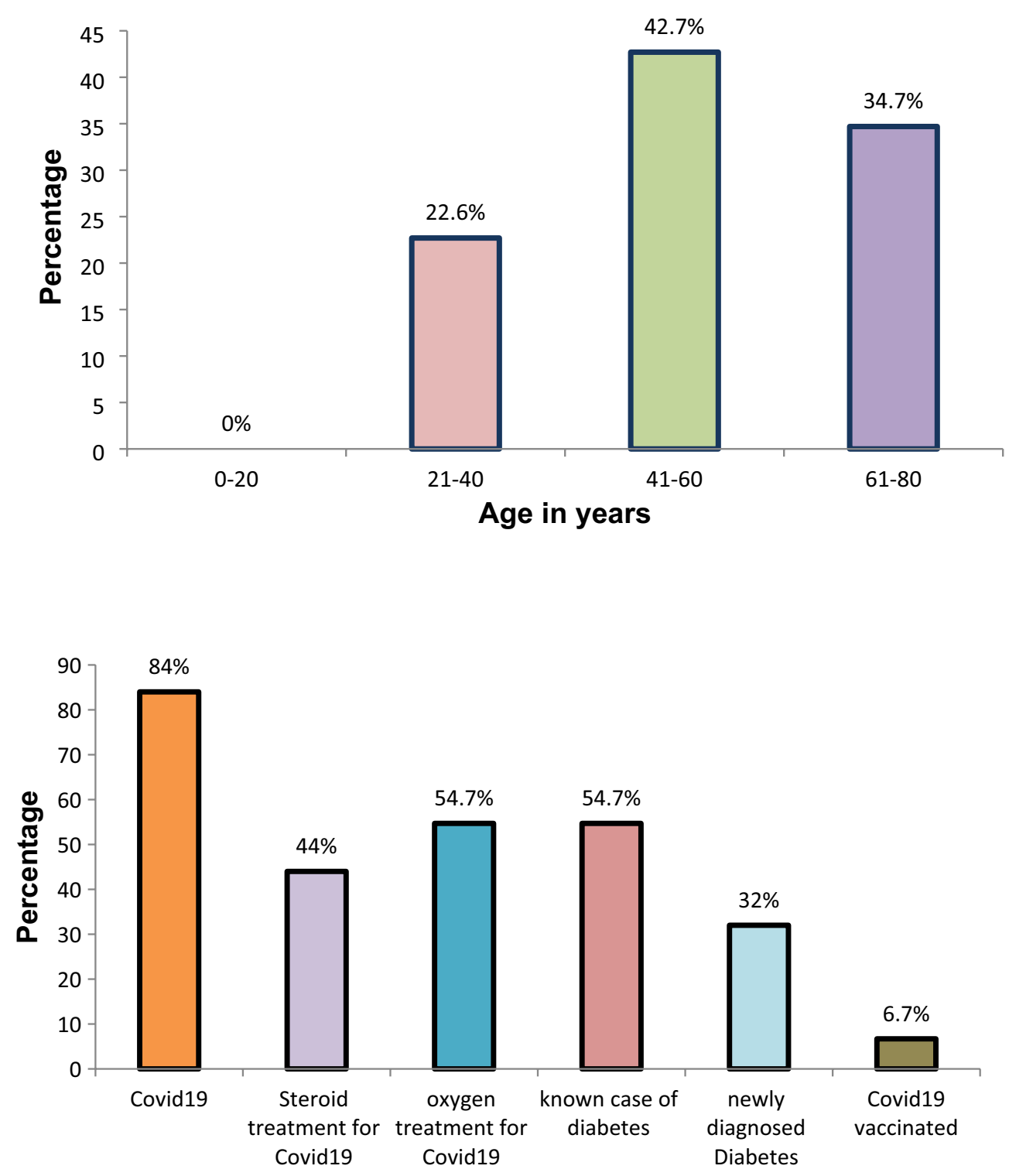

Risk factors 


\section{COVID-19 status in mucormycosis patients}

In the present study, $65.3 \%$ mucormycosis patients were postCOVID-19. $18.7 \%$ of mucormycosis patients were diagnosed during COVID-19 treatment or quarantine period (Table 2). 16\% of mucormycosis patients had no history of COVID-19 infection and were negative RTPCR results on admission.

Among mucormycosis patients with history of COVID19 infection, eight patients were with of loss of taste and smell during the COVID-19 infection. All the patients were recovered fully after few weeks and got back taste and smell sensations and no patients with mucormycosis were presented with loss of taste and smell. In the present study, no patient was infected with COVID-19 during treatment for mucormycosis.

\section{Stage of the mucormycosis at the time of admission and mortality in mucormycosis patients}

In the present study, $52 \%$ of patients were presented in stage III, $41.3 \%$ in stage II. (Table 3). In the present study, 19 patients died, $25.33 \%$ mortality was seen in mucormycosis patients.

\section{Prognosis of mucormycosis patients with diabetes mellitus}

Depending on glycemic status of patients, diabetes mellitus patients were on insulin or oral hypoglycemic drugs. Table 4 is showing prognosis of mucormycosis patients with diabetes mellitus on insulin and oral hypoglycemic drugs.

Table 1 Gap between COVID-19 treatment and symptoms of mucormycosis

\begin{tabular}{lll}
\hline $\begin{array}{l}\text { Gap between COVID-19 treatment } \\
\text { and symptoms of mucormycosis in } \\
\text { days (days) }\end{array}$ & No. of patients & Percentage (\%) \\
\hline $0-7$ & 26 & 34.7 \\
$8-15$ & 22 & 29.3 \\
$16-30$ & 12 & 16 \\
$31-45$ & 02 & 2.7 \\
$46-90$ & 01 & 1.3 \\
\hline
\end{tabular}

\section{Complications occurred during mucormycosis management}

In the present study, the frontal lobe abscess was seen in two patients, one had $10 \times 8 \mathrm{~mm}$ size and another had $35.6 \times 32 \mathrm{~mm}$ size. Two patients had temporal lobe abscess of size $9 \times 9 \mathrm{~mm}$ size and $11 \times 11 \mathrm{~mm}$ size. Six patients had cavernous sinus thrombosis. One patient had patchy meningitis. Facial nerve palsy of grade IV was seen in three patients and in all three patients taste sensations were intact.

\section{Complications due to amphotericin B}

In the present study, few patients experienced infusionrelated reactions like nausea, vomiting, chills and rigors with amphotericin deoxycholate. Long duration of the treatment also caused anemia, hypokalemia and deranged RFT in few patients. These patients were shifted to liposomal amphotericin B or pre-medicated with analgesics, antihistamines and steroids.

In the present study, thrombophlebitis was seen in $62.66 \%$ patients, fever with chills or rigors was seen in $26.66 \%$ patients, anemia was seen in $18.66 \%$ patients, deranged RFT was seen in $13.33 \%$ patients, hypokalemia was seen in $4 \%$ three patients and abdominal pain was seen in $2.66 \%$ patients. No patient in the present study had renal shutdown. No major adverse effects were seen with liposomal amphotericin B.

\section{Surgical outcome in mucormycosis patients}

In the present study, surgeries were done to remove all necrotic areas from nose, sinuses, palate, maxilla, pterygopalatine fossa, infratemporal fossa and mandible. In case of recurrence and involvement of adjacent areas, multiple surgeries were done in same patients. Orbital exenteration

Table 3 Mucormycosis stage at the time of admission

\begin{tabular}{lcl}
\hline Mucormycosis stage & No. of patients & Percentage (\%) \\
\hline I & 0 & 0 \\
II & 31 & 41.3 \\
III & 39 & 52 \\
IV & 05 & 6.7 \\
\hline
\end{tabular}

Table 2 COVID-19 status in mucormycosis patients

\begin{tabular}{lll}
\hline COVID-19 status in mucormycosis patient & No. of patients & Percentage (\%) \\
\hline Mucormycosis patients with H/o COVID-19-positive & 63 & 84 \\
Mucormycosis patients with no H/O COVID-19-positive & 12 & 16 \\
Mucormycosis during COVID-19 treatment/quarantine & 14 & 18.7 \\
Mucormycosis after COVID-19 treatment/quarantine & 49 & 65.3 \\
\hline
\end{tabular}


Table 4 Prognosis of mucormycosis patients with diabetes mellitus

\begin{tabular}{|c|c|c|c|c|}
\hline Mucormycosis patients & $\begin{array}{l}\text { No of patients } \\
\text { recovered }\end{array}$ & No of patients dead & $\begin{array}{l}\text { No of patients } \\
\text { transferred }\end{array}$ & Total \\
\hline Diabetic patients on insulin & $22(53.6 \%)$ & $18(44 \%)$ & $1(2.4 \%)$ & $41(100 \%)$ \\
\hline $\begin{array}{l}\text { Diabetic patients on oral hypo- } \\
\text { glycemic drugs }\end{array}$ & $22(91.6 \%)$ & $1(4.2 \%)$ & $1(4.2 \%)$ & $24(100 \%)$ \\
\hline Non-diabetic patients & $10(100 \%)$ & 0 & 0 & $10(100 \%)$ \\
\hline
\end{tabular}

was done by ophthalmologist in the cases with involvement of eyeball and loss of vision due to extensive mucormycosis.

In the present study, total 67 patients were operated. Six patients were not operated due to poor medical condition and died. Two patients were transferred to neurosurgery centre due to extensive disease.

Seventy-two percentage patients were recovered after surgical debridement. Although, surgical debridement was done, $17.33 \%$ patients died.

\section{Discussion}

Mucormycosis is an infection caused by Mucoraceae family and class Phygomycetes or Zygomycetes fungus [11, 12]. The fungus is commensal of the nasal mucosa. Various theories are suggested for the occurrence of mucormycosis. Diabetes mellitus, hyperglycemia in COVID-19 infection, ketoacidosis and low $\mathrm{pH}$ due to acidosis increase chances of mucormycosis. The phagocytic activity of WBC is reduced with the use of steroids, which causes defective bronchoalveolar macrophage migration, ingestion, and phagolysosome fusion, making diabetic patients prone to mucormycosis. Mucormycosis growth occurs on free available iron. Hyperglycemia causes glycosylation of transferrin, ferritin and reduces iron-binding. This increased free iron is favourable for mucormycosis growth. Increased interleukin 6 in COVID-19, increases free iron due to increased synthesis and decreased iron transport. Acidosis increases free iron by reducing the ability of transferrin to chelate iron. Adhesive bandages, wooden tongue depressors, hospital linens, negative pressure rooms, water leaks, poor air filtration, non-sterile medical devices, and building construction are sources of mucormycosis [13]. Mucormycosis is also seen in exposed wounds [14].

\section{Age and sex distribution in the study group}

According to Moorthy et al., the incidence of mucormycosis was not depending on age or gender [9]. According to Manish et al., the mean age of patients was 50.42 years and $70.97 \%$ mucormycosis patients were male [15]. According to Elzein et al., the median age of the patients was 43.45 years and $72 \%$ were males [16].
According to Bhanuprasad et al., the mean age of mucormycosis patient was 51 years, $78 \%$ were male and $22 \%$ were female [17]. According to Patel et al., 74.6\% of mucormycosis patients were men [18]. According to Singh et al. study, $78.9 \%$ of mucormycosis patients were males [19].

In the present study, $74.7 \%$ of mucormycosis patients were males and $77.4 \%$ of mucormycosis patients were above 40 years of age. In the present study, age and sex distribution among mucormycosis patients was similar to previous studies done.

\section{Risk factors associated with mucormycosis}

According to Mehta, COVID-19 is a major risk factor for mucormycosis [20]. According to Patel et al., COVID-19 was the single disease in $32.6 \%$ of COVID-19-associated mucormycosis patients [18]. In the present study, $84 \%$ of mucormycosis patients were COVID-19 positive or had history of COVID-19 infection. Present study findings are comparable to Mehta study.

According to Patel et al., 62.7\% of mucormycosis cases diabetes mellitus were most common underlying disease [18]. According to Skiada et al., diabetes was seen as a risk factor for mucormycosis in $73.5 \%$ of cases in India [1]. $90 \%$ cases of mucormycosis from North India had diabetic ketoacidosis while 10\% of cases from South India had mucormycosis [1]. It indicates that uncontrolled and not diagnosed diabetic patients were more in North India. According to Bhanuprasad, diabetes mellitus remained a significant risk factor in mucormycosis patients with and without COVID-19 infection. 97\% mucormycosis patients had underlying diabetes mellitus [17].

In the present study, diabetes mellitus was the most common risk factor seen in $86.6 \%$ of patients with mucormycosis. $54.7 \%$ of mucormycosis patients had pre-existing diabetes mellitus. $32 \%$ of mucormycosis patients were newly diagnosed with diabetes mellitus (Fig. 3). In the study done by Singh et al., pre-existing diabetes mellitus was seen in $80 \%$ of cases and among them $14.9 \%$ were in diabetic ketoacidosis. As compared to previous studies, diabetes mellitus remained most common risk factor for mucormycosis.

According to Singh et al., use of steroids for the treatment of COVID-19 was seen in 76.3\% of cases [19]. 
According to Bhanuprasad, $71.6 \%$ patients of mucormycosis had received steroids. Unwarranted steroid use even in mild COVID-19 along with other factors was responsible for the mucor epidemic in India [17]. According to Sarkar et al., a history of corticosteroid intake for the treatment of COVID-19 was present in $76.3 \%$ of cases with mucormycosis [21]. According to Pal et al., Steroid use was seen in $85 \%$ of COVID-19 associated mucormycosis cases [22].

In the present study, the history of corticosteroid intake for the treatment of COVID-19 was present in $44 \%$ of cases. In the present study, history of corticosteroid intake was seen in less number of patients compared to other studies.

According to Bhanuprasad, oxygen use was uncommon among mucormycosis cases [17]. In the present study, oxygen was given in $54.7 \%$ of patients with mucormycosis. It indicates that COVID-19 infection had lung involvement and immunosuppression had occurred due to COVID-19 infection itself.

\section{Gap between COVID-19 treatment and symptoms of mucormycosis}

According to Pal et al., the median time interval between COVID-19 diagnosis and the first evidence of mucormycosis infection was 15 days [22]. According to Muthu et al., diagnosis of mucormycosis was after a mean of 19.5 days from the diagnosis of COVID-19 [23]. 25\% of mucormycosis cases were early COVID-19 active mucormycosis, diagnosed within 7 days of COVID-19 diagnosis. Most of the mucormycosis cases in India were late COVID-19 active mucormycosis cases [23]. According to Mehta et al., 2-5 weeks gap was seen between recovery from COVID-19 and onset of symptoms of mucormycosis. According to Gerg et al., mucormycosis usually developed 10-14 days after hospitalization [24]. Mucormycosis was seen in 59.4\% active COVID-19-positive cases and 40.6\% in post-COVID-19 cases [21]. According to Patel et al., most cases of mucormycosis were diagnosed 8 or more days after COVID-19 diagnosis [18].

In the present study, $34.7 \%$ of mucormycosis cases were early COVID-19 active mucormycosis, diagnosed within 7 days of COVID-19 diagnosis. 64\% mucormycosis patients had symptoms after 15 days of COVID-19 infection. These results are similar to Garg et al. and Muthu et al. study. $80 \%$ of COVID-19-positive were presented within 1 month of COVID-19 treatment.

\section{COVID-19 status in mucormycosis patients}

According to Pal et al., history of COVID-19 was present in $37 \%$ patients with mucormycosis [22].

In the present study, $84 \%$ patient had history of COVID19 infection. $65.3 \%$ mucormycosis patients were post
COVID-19. $18.7 \%$ of mucormycosis patients were diagnosed during COVID-19 treatment or quarantine period (Table 2). $16 \%$ of mucormycosis patients had no history of COVID-19 infection. According to the present study, COVID-19 pandemic had impact for the mucurmycosis epidemic in country like India.

\section{Stage of the mucormycosis at the time of admission and mortality in mucormycosis patients}

According to Sarkar et al. [21], nose and sinus were the most common site (88.9\%), followed by rhino-orbital (56.7\%) and ROCM type (22.2\%). According to Mehta et al., ROCM type is more common in India [8]. According to Muthu et al., rhino-orbital (ROM) and rhino-orbito-cerebral mucormycosis (ROCM) were seen in $89 \%$ of cases in India and globally seen in $64 \%$ cases of mucormycosis [23]. According to Pal et al., rhino-orbital mucormycosis was seen in $42 \%$, rhinoorbito-cerebral mucormycosis was seen in $24 \%$ and pulmonary mucormycosis was seen in $10 \%$ [22]. In Patel et al. study, the rhino-orbital mucormycosis was seen in $58.2 \%$, followed by rhino-orbital-cerebral and pulmonary [18]. In the present study, $52 \%$ patients were presented in stage III followed by $41.3 \%$ in stage II. Stage II disease was limited to sino-nasal cavity and had better outcome. Data were comparable in Indian scenario.

The mortality in the patients of mucormycosis depends on the stage of mucormycosis at the time of presentation, general condition of the patient, type of fungus, and the site affected.

The delayed presentation of mucormycosis is due to a lack of clinical suspicion and difficulty in isolating fungi. As it is a time-sensitive disease, a delay of even 6 days in initiating treatment doubles the 30-day mortality from 35 to $66 \%$ [3]. According to Patel et al., mortality rates were the same in patients with COVID-19 associated mucormycosis and non-COVID-19 associated mucormycosis groups [18]. According to Aranjani, mortality due to mucormycosis had increased from 50 to $85 \%$ during the current COVID-19 pandemic [25].

According to Roden et al., mortality was $46 \%$ in only sinus infections, $76 \%$ for pulmonary infections, and $96 \%$ for disseminated mucormycosis [26]. The prognosis of mucormycosis patients is poor and the mortality ranges between 33.3 and $80 \%$ [9]. The global case fatality rate in mucormycosis is $46 \%$ [27]. The mortality in COVID-19 associated mucuormycosis was $87.5 \%$ [28-30]. Intracranial involvement of mucormycosis increases the fatality rate up to $90 \%$ [19]. Dissemination of mucormycosis is quite fast and therefore $50 \%$ of cases of mucormycosis are diagnosed after post-mortem [19]. According to Singh et al., mortality was seen in 30.7\% in COVID-19 infection with mucormycosis [19]. According to Prakash et al., the mortality rate of 
mucormycosis in India was in the range of 28-52\% [31]. According to Verma et al., the mortality rate of mucormycosis was $46 \%$ [32]. According to Pal et al., the mortality rate was 34\% in COVID-19 associated mucormycosis [22].

In the present study, mortality in mucormycosis patients was $25.33 \%$. The reason for low mortality in mucormycosis patients was because of screening of all patients in COVID hospital for mucormycosis and detection of mucormycosis in an early stage of the disease. According to Muthu et al., better survival and low mortality in mucormycosis were reported from India because of rhino-orbital cerebral mucormycosis, early intervention, the reporting bias and publication bias in these case reports.

\section{Prognosis of mucormycosis patients with diabetes mellitus}

Glucocorticoids are used for the treatment of severely ill COVID-19 patients to suppress the very high levels of cytokines and c-reactive peptides [33]. Glucocorticoids are known to cause hyperglycemia in patients with or without pre-existing diabetes. Steroids increase the blood sugar levels by increasing hepatic gluconeogenesis, increasing insulin resistance, reducing the uptake of glucose and reducing the action of Beta cells directly [34]. COVID-19, diabetes and steroid intake predisposes to mucormycosis [35]. In COVID19 infection, a direct attack on pancreatic cells expressing angiotensin-converting enzyme 2 receptors, stress hyperglycemia resulting from the cytokine storm, alterations in glucose metabolism, steroid treatment during hospitalization lead to transient hyperglycemia. 1.5-2.2\% of diabetes codes were for drug- or chemical-induced diabetes [36]. It indicates that use of the steroids is not the only factor for hyperglycemic states in COVID-19 patients. According to Bhandari et al., $44.7 \%$ of patients were known diabetics, $42.1 \%$ was newly diagnosed diabetic and $86.80 \%$ mucormycosis patients were with uncontrolled glycemic levels [35].

In the present study, $54.7 \%$ of mucormycosis patients with known diabetics, $32 \%$ were newly diagnosed with diabetes mellitus. In the present study, newly diagnosed diabetes mellitus patients were less due to low doses of steroids were given for less duration during COVID treatment.

In the present study, diabetes mellitus was the most common underlying disease in mucormycosis patients. Among diabetic patients on insulin, $44 \%$ of patients died. Among patients on oral hypoglycemic, $4.2 \%$ of patients died. The extensive disease was seen in patients with uncontrolled diabetes mellitus and mortality was increased in mucormycosis patients with uncontrolled diabetes mellitus.

\section{Complications occurred during treatment}

Complications had occurred during the treatment of mucormycosis and after aggressive surgical debridement. These complications were increased creatinine levels, osteomyelitis of bone, facial nerve palsy, brain abscess, intracranial extension of mucormycosis, cavernous sinus thrombosis, etc. Facial nerve paralysis was seen in $11 \%$ of rhino-orbital-cerebral mucormycosis [37]. According to Mehta et al., facial nerve paralysis was occasionally seen in mucormycosis epidemic. Facial nerve palsy may be the alarm for rhinocerebral mucormycosis. Facial nerve palsy occurs due to involvement of the pterygopalatine fossa, infratemporal fossa and/or premaxillary space [20].

In the present study, facial nerve palsy was seen in $4 \%$ patients with mucormycosis. These lower values were because of sino-nasal mucormycosis in $42.42 \%$ of cases. The exact aetiology for facial nerve paralysis is not known. Infection can reach from the pterygopalatine fossa to the inferior orbital fissure, orbital apex, and infratemporal fossa which lead to facial nerve palsy [37]. In diabetic patients, arterial resistance may cause oedema of the facial nerve and localized ischemia leading to facial nerve palsy distal to chorda tympani nerve. Involvement of the CNS in mucormycosis occurs due to spread from the paranasal sinuses and orbits in $70 \%$ of cases [10]. $30 \%$ of cases of CNS involvement were seen in intravenous drug users and hematogenous spread from infected distant sites. Uncommon manifestations of cranial invasion include sagittal sinus thrombosis, meningitis, obstructive hydrocephalus and epidural and subdural abscess [10].

In rhino-orbital-cerebral mucormycosis, CNS involvement is seen in 33-49\% of patients [38]. According to Therakathu et al., brain abscesses were seen in $4.65 \%$ of patients with rhino-orbital-cerebral mucormycosis [39]. In the present study, brain abscess was seen in 5.3\% of patients. $1.3 \%$ of patients had meningitis. In patients with raised serum creatinine levels had advised withhold of amphotericin B till serum creatinine came within normal limits. So no patient in the present study had renal shutdown.

\section{Complications due to amphotericin B}

Infusion-related toxicity in amphotericin B treatment is because of the pro-inflammatory response to cytokines. It is seen in the first few minutes of administration. Toxicity related to the infusion of L-AMB is less that other formulation of polyenes, including ABLC. An idiosyncratic reaction which manifested as a triad of chest pain and/or discomfort, flank and/or abdominal pain and dyspnea were seen with an infusion of L-AMB due the liposome than the active drug itself [40]. 
According to Goodwin et al., infusion-related adverse events reported were fever (51\%) and chills (28\%), followed by nausea (18\%), headache (9\%), and thrombophlebitis (5\%). After symptoms of Infusion-related adverse events reported, then patient should be premeditated for subsequent amphotericin B infusions. The most common regimens for premedication were included diphenhydramine, a corticosteroid, acetaminophen, and heparin [41].

In the present study, patients experienced infusionrelated reactions like nausea, vomiting, chills and rigors with Amphotericin deoxycholate. Long duration of the treatment also caused anemia, hypokalemia and deranged RFT in few patients. These patients were shifted to liposomal amphotericin B. Pre-medications, such as Injection Avil $2 \mathrm{cc}$, Injection Hydrocort $100 \mathrm{mg}$ intravenously and Tablet Paracetamol $500 \mathrm{mg}$, were given to the patients with infusion reactions. In the present study, thrombophlebitis was seen in 47 patients $(62.66 \%)$, fever with chills or rigors was seen in 20 patients $(26.66 \%)$, anemia was seen in 14 patients (18.66\%), deranged RFT was seen in 10 patients (13.33\%), hypokalemia was seen in three patients ( $4 \%$ ) and abdominal pain was seen in two patients (2.66\%). No patient in the present study had renal shutdown.

In the present study, thrombophlebitis was seen in more patients because of longer duration of treatment with amphotericin de-oxycholate as compared to liposomal amphotericin B. Fever with chills or rigors was seen in patients with amphotericin de-oxycholate and patients were premeditated half hour before the infusion gets started. Infusionrelated toxicity and severe idiosyncratic reaction were not seen in any patient on liposomal amphotericin B in the present study.

\section{Surgical outcome in mucormycosis patients}

Along with antifungal medications and control of underlying disease, surgical debridement is also an important treatment for mucormycosis.

In the present study, extensive surgical debridement including necrotic areas of the nasal cavity, paranasal sinuses, pterygopalatine fossa, infratemporal fossa, maxilla, mandible, palate were done. Medial maxillectomy was done to remove all necrotic tissue from maxillary sinuses, to visualize the posterior wall of the maxillary sinus and as an approach to the pterygopalatine fossa. Depending on clinical findings and CT scan or MRI as roadmap, all necrotic areas were debrided. Multiple surgeries were needed in same patient. Palatal resection, partial and total maxillectomy, mandibulectomy, orbital exenteration were also done depending on extension of the disease.

According to a study done by Ryan et al., the overall mortality ranges from 30 to $80 \%$ [42]. According to a study done by Choi et al., $82 \%$ of patients who underwent surgery were survived [43]. In the study by Abdollahi et al., 73.3\% of patients who were diagnosed early and underwent extensive surgical debridement of the infected tissues were survived [44].

In the present study, 54 patients (72\%) with mucormycosis were cured after surgical debridement. These results are comparable with Abdollahi et al. study results.

\section{Key message}

Strict glycemic control in all diabetic patients along with screenings of all COVID-19 patients for mucormycosis is must for early diagnosis and treatment.

\section{Limitations of study}

Mucormycosis cases were suddenly raised in Maharashtra (India) during second wave of COVID-19. Maximum mucurmycosis cases were reported during May 2021-July 2021 in the Institute where study was conducted. So mucormycosis cases during that period were included in study.

Funding Not applicable.

\section{Declarations}

Conflict of interest All authors declare that they have no conflict of interest.

Ethical approval All procedures performed in studies involving human participants were in accordance with the ethical standards of the institutional ethical committee as per ICMR guidelines and University protocol by letter No. GMCM/IEC-C/89/2021 and with the 1964 Helsinki Declaration and its later amendments or comparable ethical standards.

Informed consent Informed consent was obtained from all individual participants included in the study.

Consent for publication of data Informed written consent for publication of data was obtained from all individual participants included in the study.

\section{References}

1. Skiada A, Pavleas I, Drogari-Apiranthitou M (2020) Epidemiology and diagnosis of mucormycosis: an update. J Fungi 6(4):265

2. Chander J, Kaur M, Singla N et al (2018) Mucormycosis: battle with the deadly enemy over a five-year period in India. J Fungi 4(2):46. https://doi.org/10.3390/jof4020046

3. Revannavar SM, Supriya PS, Samaga L et al (2021) COVID19 triggering mucormycosis in a susceptible patient: a new 
phenomenon in the developing world? BMJ Case Rep 14:e241663. https://doi.org/10.1136/bcr-2021241663

4. Lanternier F, Dannaoui E, Morizot G et al (2012) A global analysis of mucormycosis in France: the RetroZygo study (2005-2007). Clin Infect Dis 54(1):S35-S43

5. Ibrahim AS, Spellberg B, Walsh TJ et al (2012) Pathogenesis of mucormycosis. Clin Infect Dis 54(1):S16-S22

6. Ruping MJ, Heinz WJ, Kindo AJ et al (2010) Forty-one recent cases of invasive zygomycosis from a global clinical registry. $\mathrm{J}$ Antimicrob Chemother 65(2):296-302

7. Pagano L, Valentini CG, Posteraro B et al (2009) Zygomycosis in Italy: a survey of FIMUAECMM (Federazione Italiana diMicopatologia Umana ed Animale and European Confederation of Medical Mycology). J Chemother 21(3):322-329

8. Mehta S, Pandey A (2020) Rhino-orbital mucormycosis associated with COVID-19. Cureus 12(9):e10726

9. Moorthy A et al (2021) SARS-CoV-2, uncontrolled diabetis and corticosteroids -an Unholy Trinity in invasive fungal infections of maxillofacial region? A retrospective, multicentric analysis. J Maxillofac Oral Surg 6:1-8. https://doi.org/10.1007/ s12663-021-01532-1

10. Chikley A, Ben-Ami R, Kontoyiannis DP (2019) Mucormycosis of the central nervous system. J Fungi 5(3):59. https://doi.org/10. 3390/jof5030059

11. Ferguson BJ (2000) Mucormycosis of the nose and paranasal sinuses. Otolaryngol Clin N Am 33(2):2000

12. Uckay I, Chalandon Y, Sartoretti P et al (2007) Invasive zygomycosis in transplant recipients. Clin Transplant 21:577-582

13. Chaudhry R, Venugopal P, Chopra P (1987) Prosthetic mitral valve mucormycosis caused by mucor species. Int $\mathrm{J}$ Cardiol 17(3):333-335

14. Chaves MS, Franco D, Nanni JC et al (2016) Control of an outbreak of postoperative bone mucormycosis: an intervention study of contiguous cohorts. Am J Infect Control 44(12):1715-1717

15. Manesh A, Rupali P, Sullivan MO, Mohanraj P, Rupa V, George B, Michael JS (2019) Mucormycosis-a clinicoepidemiological review of cases over 10 years. Mycoses 62(4):391-398. https://doi.org/10.1111/myc.12897 (Epub 2019 Feb 19. PMID: 30685896)

16. Elzein F, Albarrag A, Kalam K, Arafah M, Al-Baadani A, Eltayeb N, Aloteibi F, Alrashed A, Abdullah RA, Alasiri S (2020) Mucormycosis: an 8-year experience of a tertiary care centre in Saudi Arabia. J Infect Public Health 13(11):1774-1779. https://doi.org/ 10.1016/j.jiph.2020.08.002

17. Bhanuprasad K, Manesh A, Devasagayam E et al (2021) Risk factors associated with the mucormycosis epidemic during the COVID-19 pandemic. Int J Infect Dis 111:267-270

18. Patel A, Agarwal R, Rudramurthy SM, Shevkani M, Xess I, Sharma R et al (2021) Multicenter epidemiologic study of coronavirus disease-associated mucormycosis, India. Emerg Infect Dis 27(9):2349-2359. https://doi.org/10.3201/eid2709.210934. (Epub 2021 Jun 4. PMID: 34087089; PMCID:PMC8386807)

19. Singh AK, Singh R, Joshi SR, Misra A et al (2021) Mucurmycosis in COVID-19: a systematic review of cases reported worldwide and in India. Diabetes Metab Syndr Clin Res Rev 15(4):102146. https://doi.org/10.1016/j.dsx.2021.05.019

20. Mehta R, Nagarkar NM, Krishna Sasanka KSBS et al (2021) Facial nerve palsy in COVID-19-associated mucormycosis patients: a case series. Cureus 13(11):e19208. https://doi.org/10. 7759/cureus. 19208

21. Sarkar S, Gokhale T, Choudhury SS, Deb AK (2021) COVID-19 and orbital mucormycosis. Indian J Ophthalmol 69(4):1002-1004. https://doi.org/10.4103/ijo.IJO_3763_20

22. Pal R, Singh B, Bhadada SK, Banerjee M, Bhogal RS, Hage N et al (2021) COVID-19-associated mucormycosis: an updated systematic review of literature. Mycoses 64(12):1452-1459. https://doi.org/10.1111/myc.13338. (Epub2021 Jun 25. PMID: 34133798; PMCID: PMC8447126)

23. Muthu V, Rudramurthy SM, Chakrabarti A et al (2021) Epidemiology and pathophysiology of COVID-19-associated mucormycosis: India versus the rest of the world. Mycopathologia 186:739-754. https://doi.org/10.1007/s11046-021-00584-8

24. Garg D, Muthu V, Sehgal IS, Ramachandran R, Kaur H, Bhalla A, Puri GD, Chakrabarti A, Agarwal R (2021) Coronavirus Disease (COVID-19) associated mucormycosis (CAM): case report and systematic review of literature. Mycopathologia 186(2):289-298. https://doi.org/10.1007/s11046-021-00528-2 (Epub 2021 Feb 5. PMID: 33544266; PMCID: PMC7862973)

25. Aranjani JM, Manuel A, Abdul Razack HI, Mathew ST (2021) COVID-19-associated mucormycosis: evidence-based critical review of an emerging infection burden during the pandemic's second wave in India. PLoS Negl Trop Dis 15(11):e0009921. https://doi.org/10.1371/journal.pntd.0009921

26. Roden MM, Zaoutis TE, Buchanan WL et al (2005) Epidemiology and outcome of zygomycosis: a review of 929 reported cases external icon. Clin Infect Dis 41(5):634-653

27. Monte Junior ESD, Santos M, Ribeiro IB et al (2020) Rare and fatal gastrointestinal mucormycosis (Zygomycosis) in a COVID19 patient: a case report. Clin Endosc 53(6):746-749

28. Chougule A, Muthu V, Bal A et al (2015) Pulmonary gangrene due to Rhizopus spp. Staphylococcus aureus klebsiella pneumoniae and probable sarcina organisms. Mycopathologia 180(12):131-136

29. Pandey N, Kaushal V, Puri GD et al (2020) Transforming a general hospital to an infectious disease hospital for COVID-19 over 2 weeks. Front Public Health 8:382

30. Muthu V, Dhooria S, Singh Sehgal I et al (2019) The reversed halo sign and the bronchus sign: the eyes see only what the mind knows. Ann Am Thorac Soc 16(9):1203

31. Prakash H, Chakrabarti A (2021) Epidemiology of mucormycosis in India. Microorganism 9:523. https://doi.org/10.3390/micro organisms 9030523

32. Verma DK, Bali RK (2021) COVID-19 and mucormycosis of the craniofacial skeleton: causal, contributory or coincidental? J Maxillofac Oral Surg 20:165-166. https://doi.org/10.1007/ s12663-021-01547-8

33. Jin S, Hu W (2021) Severity of COVID-19 and treatment strategy for patient with diabetes. Front Endocrinol 12:469

34. Sosale A, Sosale B, Kesavadev J, Chawla M, Reddy S, Saboo B, Misra A (2021) Steroid use during COVID-19 infection and hyperglycemia-What a physician should know. Diabetes Metab Syndr 15(4):102167. https://doi.org/10.1016/j.dsx.2021.06.004. (Epub 2021 Jun 10. PMID: 34186344; PMCID: PMC8189750).

35. Bhandari S, Bhargava S, Samdhani S, Singh SN, Sharma BB, Agarwal S, Sharma MP, Sharma S, Sharma V, Kakkar S, Dube A (2021) COVID-19, diabetes and steroids: the demonic trident for mucormycosis. Indian J Otolaryngol Head Neck Surg 4:1-4

36. Barrett CE, Koyama AK, Alvarez P, et al (2022) Risk for newly diagnosed diabetes $>30$ days after SARS-CoV-2 infection among persons aged $<18$ Years-United States, March 1, 2020-June 28, 2021. MMWR Morb Mortal Wkly Rep 71:59-65. https://doi.org/ 10.15585/mmwr.mm7102e2

37. Shekar V, Sikander J, Rangdhol V, Naidu M (2015) Facial nerve paralysis: a case report of rare complication in uncontrolled diabetic patient with mucormycosis. J Nat Sci Biol Med 6(1):226-228. https://doi.org/10.4103/0976-9668.149195 (PMID: 25810669; PMCID: PMC4367044)

38. Goralska K, Blaszkowska J, Dzikowiec M (2018) Neuroinfections caused by fungi. Infection 46:443-459

39. Therakathu J, Prabhu S, Irodi A, Sudhakar SV, Yadav VK, Rupa V (2018) Imaging features of rhinocerebral mucormycosis: a study of 43 patients. Egypt J Radiol Nucl Med 49:447-452 
40. Cavassin FB, Baú-Carneiro JL, Vilas-Boas RR, Queiroz-Telles F (2021) Sixty years of amphotericin B: an overview of the main antifungal agent used to treat invasive fungal infections. Infect Dis Ther 1:1-33

41. Goodwin SD, Cleary JD, Walawander CA, Taylor JW, Grasela TH Jr (1995) Pretreatment regimens for adverse events related to infusion of amphotericin B. Clin Infect Dis 20(4):755-761

42. McSpadden RP, Martin JR et al (2017) Mucormycosis causing Ludwig angina: a unique presentation. J Oral Maxillofac Surg 75:759-762

43. Choi H, Lee H, Jeon K, Suh GY, Shin S, Kim HK, Kim K, Jeong D, Kim H (2019) Factors affecting surgical resection and treatment outcomes in patients with pulmonary mucormycosis. J Thorac Dis 11(3):892
44. Abdollahi A, Shokohi T, Amirrajab N, Poormosa R, Kasiri AM, Motahari SJ, Ghoreyshi SM, Madani SA, Nikkhah M, Ghasemi M, Larijani LV (2016) Clinical features, diagnosis, and outcomes of rhino-orbito-cerebral mucormycosis-a retrospective analysis. Curr Med Mycol 2(4):15

Publisher's Note Springer Nature remains neutral with regard to jurisdictional claims in published maps and institutional affiliations. 